\title{
Total Synthesis of Cyanolide A and Confirmation of Its Absolute Configuration
}

\section{Hyoungsu Kim and Jiyong Hong*}

Department of Chemistry, Duke University, Durham, North Carolina 27708

jiyong.hong@duke.edu

Received May 4, 2010

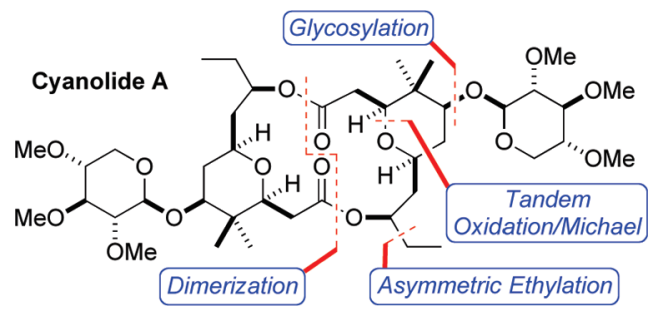

The tandem allylic oxidation/oxa-Michael reaction promoted by the gem-disubstituent effect and the 2-methyl-6-nitrobenzoic anhydride (MNBA)mediated dimerization were explored for the efficient and facile synthesis of cyanolide $A$.

Schistosomiasis (also known as bilharzia) is a chronic, debilitating parasitic disease caused by trematode flatworms of the genus Schistosoma and the second most socioeconomically devastating parasitic disease after malaria. ${ }^{1}$ More than 207 million people are infected worldwide, with an estimated 700 million people at risk in more than 70 endemic countries. $^{2}$ In sub-Saharan Africa, more than 200000 deaths per year are attributed to schistosomiasis.

Due to the lack of a vaccine, patient therapy is heavily dependent on chemotherapy with praziquantel, which currently is the only available treatment against all forms of schistosomiasis. ${ }^{3}$ Despite the success of praziquantel, relying solely on a single drug to treat 200 million people is of concern. Less than $10 \%$ of people requiring treatment are reported to obtain praziquantel. In addition, there are concerns over drug resistance and side effects. ${ }^{4}$

(1) Ross, A. G.; Bartley, P. B.; Sleigh, A. C.; Olds, G. R.; Li, Y.; Williams, G. M.; McManus, D. P. N. Engl. J. Med. 2002, 346, 1212-1220.

(2) Chitsulo, L.; Engels, D.; Montresor, A.; Savioli, L. Acta Trop. 2000, 77, 41-51.

(3) Cioli, D.; Pica-Mattoccia, L. Parasitol. Res. 2003, 90, S3-9.

(4) Melman, S. D.; Steinauer, M. L.; Cunningham, C.; Kubatko, L. S.; Mwangi, I. N.; Wynn, N. B.; Mutuku, M. W.; Karanja, D. M. S.; Colley, D. G.; Black, C. L.; Secor, W. E.; Mkoji, G. M.; Loker, E. S. PLoS. Negl. Trop. Dis. 2009, 3, e504.
An even greater challenge to schistosomiasis control is the complex lifecycle of the parasite that requires both an aquatic snail vector and a mammalian host to complete their reproductive cycle. ${ }^{1}$ Snails such as the genus Biomphalaria act as intermediate hosts in the life cycle of the parasitic trematodes. As a result, eradicating the disease in the mammalian host with a drug like praziquantel does not protect against reinfection from recurring exposure to water containing snail hosts. Therefore, molluscicides have been considered the most promising means to break the transmission cycle of the parasite. ${ }^{5}$ Niclosamide is the most widely used molluscicide available and effectively kills snails at all stages of the lifecycle. ${ }^{6}$ However, it possesses major disadvantages, including high cost, low water solubility and dispersibility, and hazardous environmental effects. As a result, considerable effort has been devoted to the identification of novel molluscicides, ${ }^{7}$ but none have proved effective enough to substitute niclosamide.

Recently, Gerwick and co-workers used a simple molluscicidal bioassay to screen marine cyanobacterial extracts and

(5) Perrett, S.; Whitfield, P. J. Parasitol. Today 1996, 12, 156-159. 295.

(7) Whitfield, P. J. Trans. R. Soc. Trop. Med. Hyg. 1996, 90, 596-600. 
Scheme 1. Retrosynthetic Plan for Cyanolide A (1)
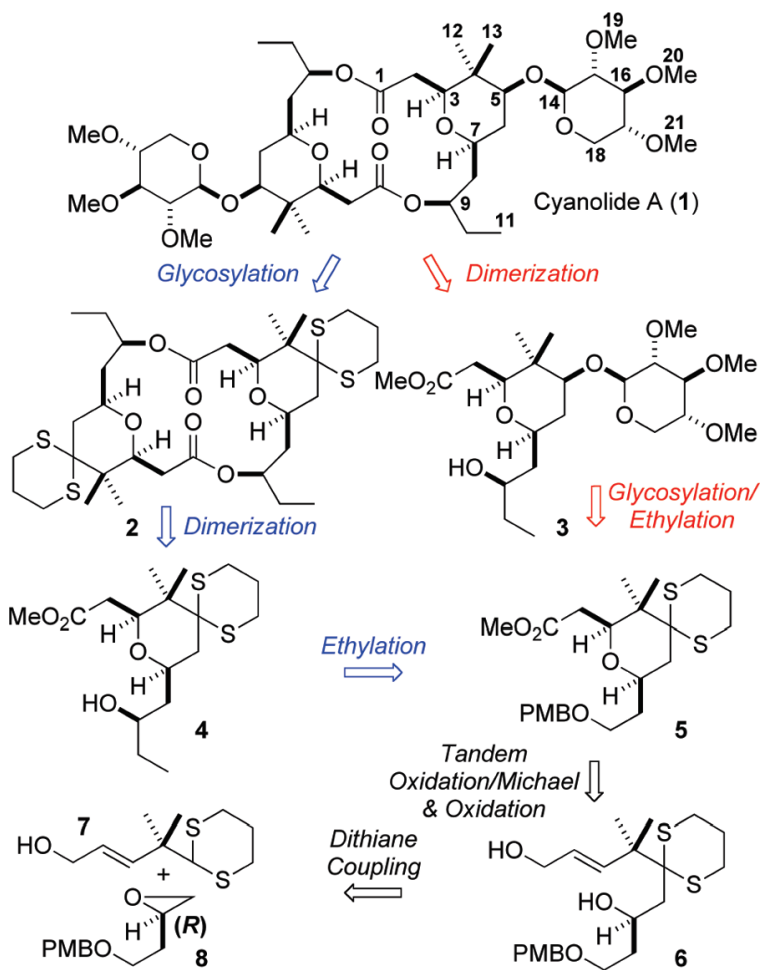

reported the isolation and structure elucidation of cyanolide A (1, Scheme 1), a new molluscicidal agent obtained from a Papua New Guinea collection of Lyngbya bouillonii. ${ }^{8}$ Compound $\mathbf{1}$ is a dimeric glycosidic macrolide consisting of a central 16-membered macrocycle fused with two tetrahydropyrans and two xylose residues. The structure and relative stereochemistry of $\mathbf{1}$ were determined by extensive NMR spectroscopic analysis, but the absolute configuration of 1 was tentatively assigned on the basis of a natural D-configuration for the xylose residue and its structural similarities to clavosolides A-D. ${ }^{9}$ It exhibited highly potent molluscicidal activity against Biomphalaria glabrata $\left(\mathrm{LC}_{50}\right.$ $=1.2 \mu \mathrm{M})$ but showed modest brine shrimp toxicity $\left(\mathrm{LC}_{50}\right.$ $=10.8 \mu \mathrm{M})$.

Due to both the great potential as a promising molluscicidal compound for controlling schistosomiasis-carrying snails of the genus Biomphalaria and the scarcity $(0.1 \%$ isolation yield) of cyanolide A (1), we sought to develop an efficient and facile synthetic route that would be amenable to the synthesis of gram-scale quantities of $\mathbf{1}$ and also to the synthesis of analogues for further biological studies. Herein, we report two efficient, facile, and complementary routes to 1 through a tandem allylic oxidation/oxa-Michael reaction promoted by the gem-disubstituent effect and 2-methyl-6nitrobenzoic anhydride (MNBA)-mediated dimerization.

(8) Pereira, A. R.; McCue, C. F.; Gerwick, W. H. J. Nat. Prod. 2010 $73,217-220$

(9) (a) Rao, M. R.; Faulkner, D. J. J. Nat. Prod. 2002, 65, 386-388. (b) Erickson, K. L.; Gustafson, K. R.; Pannell, L. K.; Beutler, J. A.; Boyd, M. R. J. Nat. Prod. 2002, 65, 1303-1306.
Scheme 1 summarizes our approach for the stereoselective synthesis of cyanolide A (1) from the readily available 1,3dithiane $\mathbf{7}$ and chiral epoxide 8. Since there were reports that final glycosylation reactions of structurally similar substrates with xylose derivatives generally gave the possible $\alpha, \alpha$ - and $\alpha, \beta$-anomers in addition to the desired $\beta, \beta$-anomer with low diastereoselectivities and yields, ${ }^{10}$ we decided to explore two complementary routes to $\mathbf{1}$, dimerization-glycosylation and glycosylation-dimerization strategies. In the dimerizationglycosylation route, we envisioned that $\mathbf{1}$ could be assembled by a glycosylation of macrolide $\mathbf{2}$ with a xylose derivative. Macrolide $\mathbf{2}$ could be prepared by an asymmetric ethylation of 2,6-cis-tetrahydropyran methyl ester $\mathbf{5}$ followed by a dimerization of hydroxy ester 4 . In the glycosylation-dimerization route, the synthesis of $\mathbf{1}$ could be accomplished by a dimerization of monomer $\mathbf{3}$ appendaged with the xylose moiety. Monomer $\mathbf{3}$ could be prepared by glycosylation followed by asymmetric ethylation of 2,6-cis-tetrahydropyran methyl ester 5. The tandem allylic oxidation/oxa-Michael reaction of diol $\mathbf{6}$, which was previously reported by our group, ${ }^{11}$ was anticipated to stereoselectively provide the key intermediate 2,6-cis-tetrahydropyran methyl ester $\mathbf{5}$.

Scheme 2. Tandem Oxidation/Oxa-Michael and NHC-Catalyzed Oxidation To Give the THP Ester $\mathbf{5}$

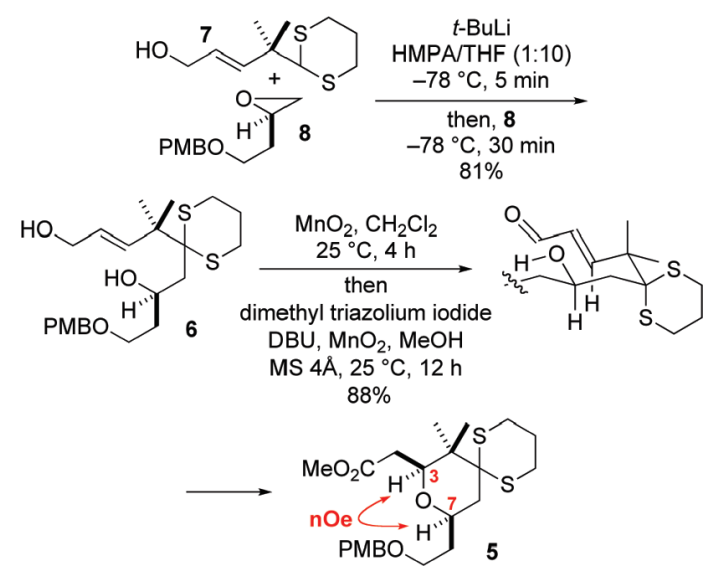

The synthesis of cyanolide A (1) started with the preparation of diol 6 for tandem reaction (Scheme 2). The coupling of the known 1,3-dithiane $\mathbf{7}^{12}$ and chiral epoxide $\mathbf{8}^{13}$ smoothly

(10) (a) Barry, C. S.; Bushby, N.; Charmant, J. P. H.; Elsworth, J. D.; Harding, J. R.; Willis, C. L. Chem. Commun. 2005, 5097-5099. (b) Son, J. B.; Kim, S. N.; Kim, N. Y.; Lee, D. H. Org. Lett. 2006, 8, 661-664. (c) Smith, A. B., III.; Simov, V. Org. Lett. 2006, 8, 3315-3318. (d) Barry, C. S.; Elsworth, J. D.; Seden, P. T.; Bushby, N.; Harding, J. R.; Alder, R. W.; Willis, C. L. Org. Lett. 2006, 8, 3319-3322. (e) Chakraborty, T. K.; Reddy, V. R.; Chattopadhyay, A. K. Tetrahedron Lett. 2006, 47, 74357438 .

(11) Kim, H.; Park, Y.; Hong, J. Angew. Chem., Int. Ed. 2009, 48, 75777581 .

(12) (a) Armesto, D.; Horspool, W. M.; Gallego, M. G.; Agarrabeitia, A. R. J. Chem. Soc., Perkin Trans. 1 1992, 163-169. (b) Taylor, E. C.; LaMattina, J. L. Tetrahedron Lett. 1977, 18, 2077-2080.

(13) (a) Nielsen, L. P. C.; Stevenson, C. P.; Blackmond, D. G.; Jacobsen, E. N. J. Am. Chem. Soc. 2004, 126, 1360-1362. (b) Mohapatra, D. K.; Das, P. P.; Reddy, D. S.; Yadav, J. S. Tetrahedron Lett. 2009, 50, 59415944. 
proceeded to set the stage for the key tandem allylic oxidation/ oxa-Michael reaction. The one-pot allylic oxidation/oxa-Michael/ oxidation of $6\left(\mathrm{MnO}_{2}, \mathrm{CH}_{2} \mathrm{Cl}_{2}, 25{ }^{\circ} \mathrm{C}, 4 \mathrm{~h}\right.$, then dimethyl triazolium iodide, $\mathrm{MnO}_{2}, \mathrm{DBU}, \mathrm{MeOH}, \mathrm{MS} 4 \AA, 25{ }^{\circ} \mathrm{C}$, $12 \mathrm{~h})^{11,14}$ afforded the desired 2,6-cis-tetrahydropyran methyl ester $5(88 \%)$ as a single diastereomer without a trace of the diastereomeric 2,6-trans-tetrahydropyran methyl ester. The relative stereochemistry of the 2,6-disubstituted tetrahydropyran 5 was determined to be cis by extensive 2D NOESY study (see the Supporting Information). The excellent stereoselectivity observed in the oxa-Michael reaction was probably due to a highly rigid chairlike transition state induced by "double gem-disubstituent effects" of C4-gemdimethyl and C5-1,3-dithiane groups. Importantly, the sequence of 1,3-dithiane coupling and tandem reaction was highly stereoselective, operationally simple, and robust, and it could be performed on a gram-scale.

Having established an efficient and facile synthetic route to gram-quantities of the key intermediate 2,6-cis-tetrahydropyran 5, the dimerization-glycosylation route was first explored (Scheme 3). To stereoselectively install the C9hydroxyl group, we investigated the utility of 3-exo-morpholinoisoborneol (MIB)-catalyzed asymmetric addition of $\mathrm{Et}_{2} \mathrm{Zn} .{ }^{15}$ Deprotection of the PMB group in $\mathbf{5}$ followed by Parikh-Doering oxidation of the resulting alcohol 9 provided aldehyde 10. Asymmetric ethylation of $\mathbf{1 0}$ in the presence of $\mathrm{Et}_{2} \mathrm{Zn}$ and (+)-MIB provided the desired secondary alcohol 4 in $86 \%$ yield $(\mathrm{dr}=7: 1) .{ }^{16}$ The configuration of the C9 stereocenter was assigned as $(R)$ using Kakisawa's extension of Mosher's method ${ }^{17}$ and later confirmed by single-crystal X-ray analysis of $\mathbf{1 1}$.

With monomeric unit $\mathbf{4}$ in hand, we turned our attention to dimerization to complete the synthesis of $\mathbf{1}$. Recently, in a related transformation, we found that Shiina's lactonization protocol with $\mathrm{MNBA}^{18}$ appeared to be an excellent method for macrolactonization owing to its remarkable efficiency and simple operation. ${ }^{11,19}$ Hydrolysis of $\mathbf{4}$ under basic conditions followed by dimerization of the corresponding hydroxy carboxylic acid (MNBA, DMAP, toluene, $90{ }^{\circ} \mathrm{C}, 12 \mathrm{~h}$ ) smoothly proceeded to provide dimeric macrolide $2(53 \%$ for two steps). Deprotection of the 1,3-dithiane group and $\mathrm{NaBH}_{4}$-reduction of the corresponding ketone completed the synthesis of cyanolide A aglycone $\mathbf{1 1}$.

After exploring a number of glycosylation conditions, glycosylation of $\mathbf{1 1}$ with phenyl thioglycoside $\mathbf{1 2}^{10 \mathrm{a}}$ in the presence of $\mathrm{MeOTf}^{20}$ proceeded smoothly to give the desired $\beta, \beta$-anomeric cyanolide A (1) (21\%) along with $\beta, \alpha-$

(14) Maki, B. E.; Scheidt, K. A. Org. Lett. 2008, 10, 4331-4334.

(15) (a) Nugent, W. A. Chem. Commun. 1999, 1369-1370. (b) Chen, Y. K.; Lurain, A. E.; Walsh, P. J. J. Am. Chem. Soc. 2002, 124, 1222512231.

(16) (-)-MIB-catalyzed asymmetric ethylation of $\mathbf{1 0}$ afforded C9-epi $\mathbf{4}$ with a higher diastereoselectivity $(\mathrm{dr}>20: 1)$.

(17) Ohtani, I.; Kusumi, T.; Kashman, Y.; Kakisawa, H. J. Am. Chem. Soc. 1991, 113, 4092-4096.

(18) (a) Shiina, I.; Kubota, M.; Oshiumi, H.; Hashizume, M. J. Org. Chem. 2004, 69, 1822-1830. (b) Shiina, I.; Fukui, H.; Sasaki, A. Nat. Protoc. 2007, 2, 2312-2317.

(19) (a) Shiina, I.; Katoh, T.; Nagai, S.; Hashizume, M. Chem. Rec. 2009, 9, 305-320. (b) Schäckel, R.; Hinkelmann, B.; Sasse, F.; Kalesse, M. Angew. Chem., Int. Ed. 2010, 49, 1619-1622.
Scheme 3. Total Synthesis of Cyanolide A (1) via Dimerization-Glycosylation Strategy
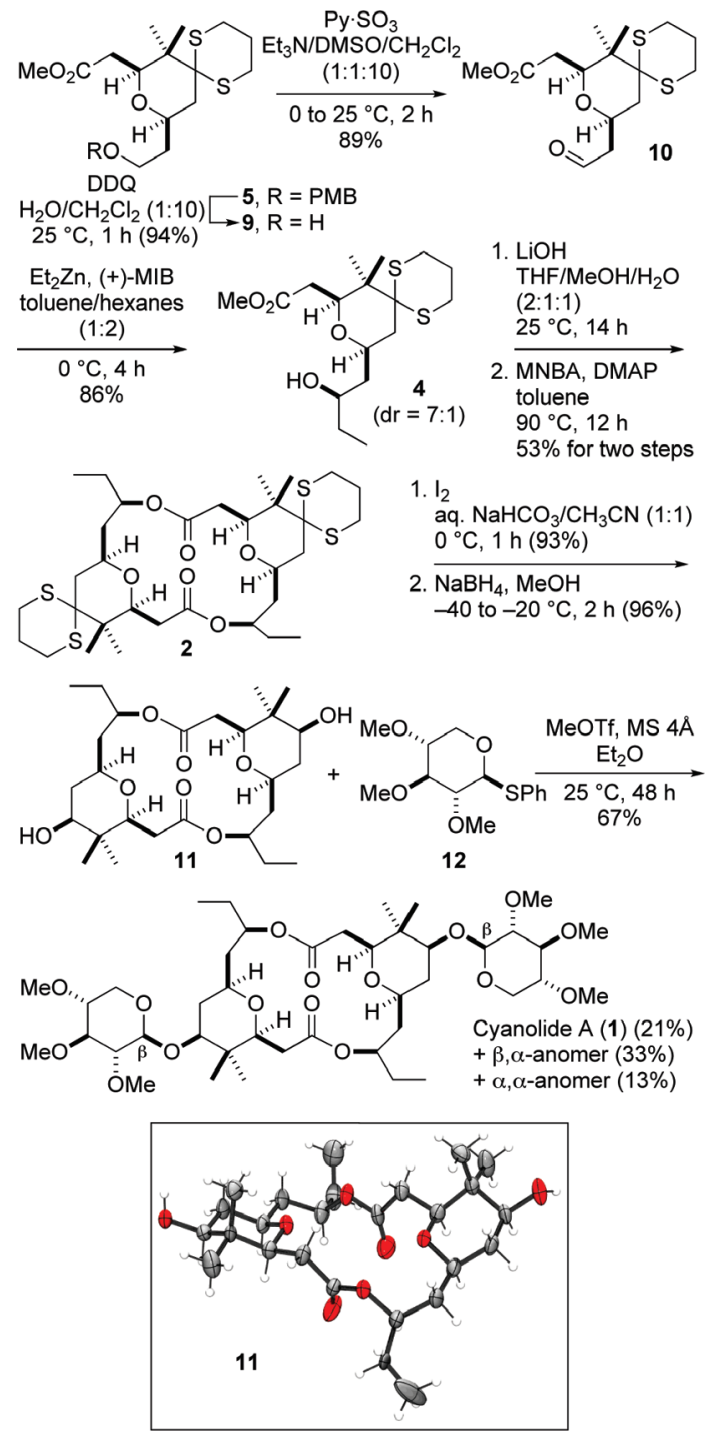

anomeric (33\%) and $\alpha, \alpha$-anomeric (13\%) isomers. The low yield and stereoselectivity of the glycosylation reaction have been observed with structurally similar substrates. ${ }^{10}$ The synthetic cyanolide A (1) proved identical in all respects with the authentic natural product. $^{8}$ The optical rotation of our synthetic $\mathbf{1}\left([\alpha]^{25} \mathrm{D}-55.5, c 0.33, \mathrm{CHCl}_{3}\right)$ was in agreement with that of the natural $1\left([\alpha]^{23}{ }_{\mathrm{D}}-59, c 0.6, \mathrm{CHCl}_{3}\right)$, confirming the absolute stereochemistry of $\mathbf{1}$ proposed by Gerwick and co-workers. ${ }^{8}$

Even though the dimerization-glycosylation route successfully completed the synthesis of $\mathbf{1}$, we thought that the final glycosylation step was not stereoselective and efficient enough for the generation of a variety of carbohydrate analogues for biological studies. To establish a more efficient method for the

(20) (a) Lönn, H. J. Carbohydr. Chem. 1987, 6, 301-306. (b) Watanabe, H.; Nakada, M. J. Am. Chem. Soc. 2008, 130, 1150-1151. NBS-promoted glycosylation of $11\left(\mathrm{NBS}, \mathrm{CH}_{3} \mathrm{CN}\right.$, MS $4 \AA,-40$ to $\left.0{ }^{\circ} \mathrm{C}, 2 \mathrm{~h}\right)$ gave a lower stereoselectivity. 
Scheme 4. Total Synthesis of Cyanolide A (1) via Glycosylation-Dimerization Strategy

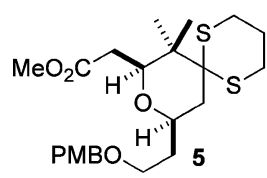

1. $\mathrm{I}_{2}$

aq. $\mathrm{NaHCO}_{3} / \mathrm{CH}_{3} \mathrm{CN}(1: 1)$

$0{ }^{\circ} \mathrm{C}, 1 \mathrm{~h}(95 \%)$

2. $\mathrm{NaBH}_{4}, \mathrm{MeOH}$

-40 to $-20^{\circ} \mathrm{C}, 1 \mathrm{~h}$

quantitative
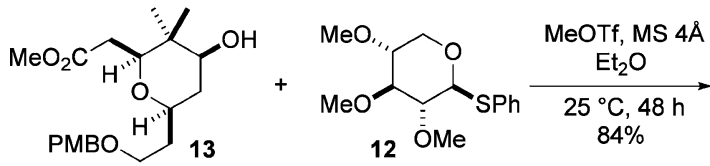

12 ÖMe

$84 \%$

1. DDQ, $\mathrm{H}_{2} \mathrm{O} / \mathrm{CH}_{2} \mathrm{Cl}_{2}(1: 10)$

$25^{\circ} \mathrm{C}, 1 \mathrm{~h}(91 \%)$
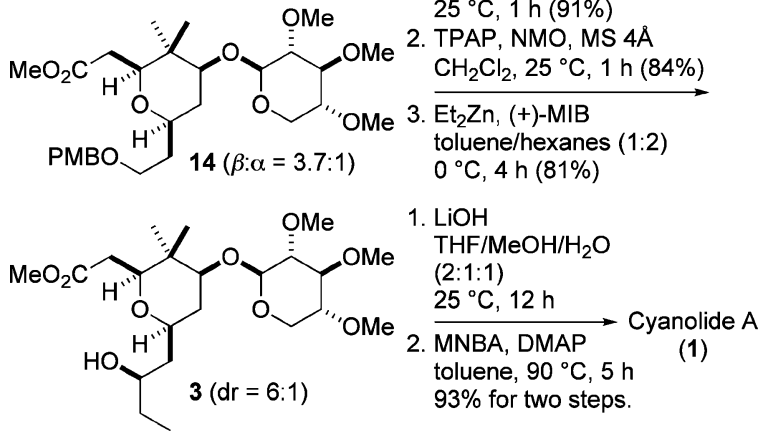

installation of the xylose moiety, we investigated an alternative glycosylation-dimerization route (Scheme 4).

The key intermediate 2,6-cis-tetrahydropyran 5 was converted to secondary alcohol $\mathbf{1 3}$ by deprotection of the 1,3dithiane group in $\mathbf{5}$ followed by $\mathrm{NaBH}_{4}$-reduction of the corresponding ketone. ${ }^{21}$ Glycosylation of $\mathbf{1 3}$ with phenyl thioglycoside $\mathbf{1 2}$ in the presence of MeOTf smoothly proceeded to afford the desired $\beta$-anomeric monomer 14 (66\%) along with $\alpha$-anomeric monomer $(18 \%){ }^{22}$ We were pleased to observe the improved stereoselectivity in the glycosylation step $(1.3: 1$ to $3.7: 1){ }^{23}$

(21) The relative stereochemistry at the C5-position in $\mathbf{1 3}$ was determined by $2 \mathrm{D}$ NMR analysis of its corresponding acetate (see the Supporting Information).

(22) NBS-promoted glycosylation of $\mathbf{1 3}$ (NBS, $\mathrm{CH}_{3} \mathrm{CN}, \mathrm{MS} 4 \AA$ A, -40 to $0{ }^{\circ} \mathrm{C}, 2 \mathrm{~h}$ ) gave a complex reaction mixture due to partial deprotection of $\mathrm{PMB}$ group under the reaction conditions.
PMB-deprotection of 14, TPAP-oxidation, and (+)-MIBcatalyzed asymmetric addition of $\mathrm{Et}_{2} \mathrm{Zn}$ afforded the desired C9 $(R)$ secondary alcohol 3 with good diastereoselectivity (dr $=6: 1,81 \%$ ). Hydrolysis of the methyl ester group in $\mathbf{3}$ and MNBA-mediated dimerization of the resulting hydroxy carboxylic acid provided cyanolide A (1) with excellent yield (93\% for two steps). The chemoselective nature of tandem allylic oxidation/oxa-Michael reaction, the efficiency of dithiane coupling reactions, and the improved stereoselectivity in the glycosylation step enabled an efficient and facile synthesis of cyanolide A (1) (10 steps, 22\% overall yield from the readily available known 7 and 8 ).

In summary, we completed the stereoselective synthesis of cyanolide A (1) from the readily available 1,3-dithiane 7 and chiral epoxide $\mathbf{8}$ through two complementary routes, dimerization-glycosylation and glycosylation-dimerization. We applied a tandem allylic oxidation/oxa-Michael reaction of 6 to the synthesis of 2,6-cis-tetrahydropyran 5. For the dimerization step, we explored the MNBA-mediated lactonization protocol to demonstrate its high efficiency and simple operation. The glycosylation-dimerization route proved more efficient for the synthesis of $\mathbf{1}$. Comparison of synthetic $1\left([\alpha]^{25}\right)$ with natural 1 enabled the confirmation of the absolute stereochemistry of the natural product. These synthetic routes would be broadly applicable to the efficient synthesis of a diverse set of cyanolide A (1) analogues for further biological studies.

Acknowledgment. This work was supported by Duke University. We are grateful to Marina Dickens for the X-ray crystal structure determination and the NCBC (Grant No. 2008-IDG-1010) for funding of NMR instrumentation.

Supporting Information Available: General experimental procedures including spectroscopic and analytical data for 1-7, 9, 11, 13, and 14 along with copies of ${ }^{1} \mathrm{H}$ and ${ }^{13} \mathrm{C}$ NMR spectra; detailed assay procedure. This material is available free of charge via the Internet at http://pubs.acs.org.

\section{OL101022Z}

(23) (a) Seden, P. T.; Charmant, J. P. H.; Willis, C. L. Org. Lett. 2008, 10, 1637-1640. (b) Chakraborty, T. K.; Reddy, V. R.; Gajula, P. K. Tetrahedron 2008, 64, 5162-5167. 